\title{
CrystEngComm
}

Check for updates

Cite this: CrystEngComm, 2017, 19, 6883

Received 13th August 2017,

Accepted 30th October 2017

DOI: 10.1039/c7ce01481c

rsc.li/crystengcomm

\section{Structures and phase transitions in neat 4,4'-di- tert-butyl-2,2'-bipyridyl and in its molecular complexes with either bromanilic or iodanilic acid $\dagger$}

\author{
M. Rok, iD *a P. Szklarz, ${ }^{a}$ M. Moskwa, ${ }^{a}$ M. Kijewska, ${ }^{a}$ J. Baran, ${ }^{b}$ G. Bator, ${ }^{a}$ \\ W. Medycki ${ }^{\mathrm{C}}$ and M. Zamponi ${ }^{\mathrm{d}}$
}

\begin{abstract}
Using the DSC method, structural phase transitions have been found at 165 and $219 \mathrm{~K}$ for 4,4'-di-tertbutyl-2,2'-bipyridyl (dtBBP), whereas for its complex with iodanilic acid (dtBBP.IA), its phase transition is at $331 \mathrm{~K}$. For the other molecular complex described (with bromanilic acid, dtBBP.BRA), no phase transitions have been observed between the liquid nitrogen temperature and the temperature of crystal decomposition. The crystal structures of the neat dtBBP have been determined at 130, 190 and $250 \mathrm{~K}$, whereas for its molecular complex with bromanilic acid (BRA) is determined at $150 \mathrm{~K}$ and for its complex with iodanilic acid (IA) determined at 293 and $345 \mathrm{~K}$. The crystal structure of neat dtBBP is characterized by layers of molecules, which are grouped by their $t$-butyls. The structures of dtBBP.BRA and dtBBP.IA are analogous to one another and consist of infinite chains of alternating acid and base molecules linked by hydrogen bonds. On the basis of the structural, dielectric and spectroscopic results (infrared and Raman), the mechanisms of the phase transitions have been proposed for dtBBP and dtBBP.IA. The ${ }^{1} \mathrm{H}$ NMR spin-lattice relaxation times for all compounds under study, as well as the second moment for the neat dtBBP in a wide temperature, have been measured. The anomalies in the temperature dependent infrared spectra together with the NMR results support the supposition that for dtBBP the dynamics of tert-butyl groups is responsible for the mechanism of the phase transitions found. The dynamics of the molecules in the crystals presented is related to the rotation both of the methyl and tert-butyl groups.
\end{abstract}

\section{Introduction}

Molecular complexes are formed from base (proton acceptor, B) and acid (proton donor, A) molecules. These molecules are usually linked by hydrogen bonds (h.b.) in their solid state, creating a variety of structures of different stoichiometries. Among others, the infinite chains of alternating acid and base $[\mathrm{A} \cdots \mathrm{B} \cdots \mathrm{A} \cdots \mathrm{B}]_{\infty}$ molecules have been frequently found. The chain formation is strongly desired from the viewpoint of

\footnotetext{
${ }^{a}$ Faculty of Chemistry, University of Wroclaw, 14 F. Joliot - Curie, 50-383 Wroclaw, Poland. E-mail: magdalena.rok@chem.uni.wroc.pl

${ }^{b}$ Institute of Low Temperature and Structure Research, Polish Academy of Science, Okólna 2, PO Box 937, 50-950 Wroclaw, Poland

${ }^{c}$ Institute of Molecular Physics, Polish Academy of Sciences, Smoluchowskiego 17, 60-179 Poznań, Poland

${ }^{d}$ Jülich Centre for Neutron Science (JCNS) at Heinz Maier-Leibnitz Zentrum

(MLZ), Forschungszentrum Jülich GmbH, Lichtenbergstr. 1, 85748 Garching; Germany

$\dagger$ Electronic supplementary information (ESI) available. CCDC 1567116-1567121. For ESI and crystallographic data in CIF or other electronic format see DOI: $10.1039 / \mathrm{c} 7 \mathrm{ce} 01481 \mathrm{c}$
}

the interesting dielectric properties of these organic materials. We can more or less predict the behaviour of protons between acid and base molecules based on the $\mathrm{p} K_{\mathrm{a}}$ value. In solution, the proton is normally present on the molecule with the higher $\mathrm{p} K_{\mathrm{a}}$ value. The difference in $\mathrm{p} K_{\mathrm{a}}\left[\Delta \mathrm{p} K_{\mathrm{a}}=\mathrm{p} K_{\text {abase }}-\right.$ $\left.\mathrm{p} K_{\mathrm{aacid}}\right]$ of the carboxylic acid and the aromatic base is a great indicator in order to determine whether a hydrogen bond exists with or without proton transfer. It was suggested that the possibility of proton transfer increases when the $\mathrm{p} K_{\mathrm{a}}$ values of the participating molecules match. In the pharmaceutical industry, the rule of thumb for salt formation is a $\mathrm{p} K_{\mathrm{a}}$ difference greater than 2 or 3 and this is typically used as a criterion for selecting counterions for salt formation. ${ }^{1}$ Johnson and Rumon, ${ }^{2}$ however, suggested limits. They have been reported that $\Delta \mathrm{p} K_{\mathrm{a}}<3.75$ affords a neutral hydrogen bond, whereas $\Delta \mathrm{p} K_{\mathrm{a}}>3.75$ results in proton transfer. They have pointed out that in the series of complexes composed of carboxylic acids and pyridines, there exists a critical region of $\Delta \mathrm{p} K_{\mathrm{a}}$ of interacting components. They suggested that protons are then located close to the centre of the bridge with single minimum potential for the proton motion. On the other 
hand, Cruz-Cabeza calculated the $\Delta \mathrm{p} K_{\mathrm{a}}$ values for a large and varied set of acid-base multicomponent crystal structures (6465) in the Cambridge Structural Database. ${ }^{3}$ The results have been divided into three zones: zone $1\left(\Delta \mathrm{p} K_{\mathrm{a}}<-1\right)$, zone $2\left(-1 \leq \Delta \mathrm{p} K_{\mathrm{a}} \leq 4\right)$ and zone $3\left(\Delta \mathrm{p} K_{\mathrm{a}}>4\right)$. The ionized acidbase complexes are observed exclusively for $\Delta \mathrm{p} K_{\mathrm{a}}>4$ and non-ionised acid-base complexes are observed for $\Delta \mathrm{p} K_{\mathrm{a}}<-1$.

If one selects the appropriate difference in the acid-base equilibrium constants, $\Delta \mathrm{p} K_{\mathrm{a}}$, the hydrogen bond is strong enough to exhibit the proton dynamics and jumps over a barrier between the proton donor and acceptor centres, even at room temperature. ${ }^{4}$ This phenomenon results in the semiconducting properties, when the alternating current, $a c$, is applied. These properties reveal a strong relationship of the ac conductivity on temperature and frequency. Herein, the $\mathrm{p} K_{\mathrm{a}}$ value of 4,4'-di-tert-butyl-2,2'-bipyridyl equals 5.11 , while the $\mathrm{p} K_{\mathrm{a} 1}$ of chloranilic acid is 0.76 . The difference in $\mathrm{pKa}$ $\left(\Delta \mathrm{p} K_{\mathrm{a}}=4.35\right)$ suggests the creation an ionic form rather than the neutral one.

Molecular complexes with hydrogen bonds have been widely investigated. ${ }^{5-11}$ The semiconducting properties of organic compounds seem interesting from the viewpoint of crystal engineering. For example, Horiuchi et al. studied phenazine and 2,5-dichloro-3,6-dihydroxy- $p$-benzoquinone (chloranilic acid) linked via chains of hydrogen bonds or 5,5'dimethyl-2,2'-bipyridyl with chloranilic acid, showing a proton transfer mediated antiferroelectric phase transition. ${ }^{12-15}$ The mechanism of this transition is governed by the proton jumping along the OHN hydrogen bond chains. On one side of the 5,5'-dimethyl-2,2'-bipyridyl molecule, the proton is transferred from the acid, $\mathrm{N}^{+}-\mathrm{H}^{\cdots} \mathrm{O}^{-}$, while on the other one it is not, $\mathrm{O}-\mathrm{H} \cdots \mathrm{N}$.

The other systems, however, composed of analogous base molecules, 4,4'- and 6,6'-dimethyl-derivatives, did not show such a property. In their case, this property was discussed rather in terms of a marked elongation of the OHN hydrogen bonds. The shortest h.b. (2.569 $\AA$ ) has been found just for the $5,5^{\prime}$-dimethyl derivative on both sides of the bipyridyl molecule. According to Table S1 (ESI $\dagger$ ), those values suggest that a hydrogen bond can be classified as a strong one. Nevertheless, the former complexes showed interesting dielectric properties as well. ${ }^{16}$

The other interesting feature of the molecular complexes is a possible $\pi-\pi$ stacking arrangement of molecules. For example, 2,5-dichloro-3,6-dihydroxy-p-benzoquinone (chloranilic acid, CLA) forms in the crystalline state with 4,4'di-tert-butyl-2,2'-bipyridyl (dtBBP) infinite hydrogen-bonded chains along the crystallographic $b$-axis. ${ }^{17}$ The molecules are linked by the $\pi-\pi$ stacking interactions as well. The hydrogen bonds found can be either with the proton transfer, $\mathrm{N}^{+}-$ $\mathrm{H}^{\cdots} \mathrm{O}^{-}$, or without, $\mathrm{O}-\mathrm{H} \cdots \mathrm{O}$. The chains of hydrogen bonds are elongated antiparallel in the crystal structure. The $\mathrm{N}^{+}-$ $\mathrm{H}^{\cdots} \mathrm{O}^{-}$hydrogen bond length is very short $(2.615 \AA)$, but a little longer than that in the antiferroelectric complex of CLA with 5,5'-dimethyl-2,2'-bipyridyl. A weak, close to continuous, phase transition has been found at $412 \mathrm{~K}$ (cooling/ heating) for the dtBBP.CLA complex. The mechanism of the phase transition has been proposed by Rok et $a l^{17,18}$ (Scheme 1). It was shown that it is related to the proton disorder in the hydrogen bonds. The dielectric permittivity of dtBBP.CLA revealed a strong anisotropy $\left(\varepsilon^{\prime}=120\right.$ and 20 along the $b$ and $c$-axes ( $412 \mathrm{~K}$ ), respectively). At low frequencies, the ac electric conductivity of the crystal has been ascribed to an activation process, while at high frequencies, the dielectric relaxation has been found to be of the ColeCole type.

All these experimental observations for the organic complexes led us to synthesize and characterize the other complexes of dtBBP. One is the bromo- and the other one is the iodo-derivative of 3,6-dihydroxy-p-benzoquinone, i.e. 2,5dibromo-3,6-dihydroxy- $p$-benzoquinone (bromanilic acid, BRA) and 2,5-diiodo-3,6-dihydroxy- $p$-benzoquinone (iodanilic acid, IA). In this paper, we will present the crystal structures, spectroscopic and dielectric properties of the dtBBP base and its complexes with either bromanilic or iodanilic acid. The mechanism of the structural phase transition in dtBBP.IA will be discussed as well.

\section{Experimental section}

\subsection{Materials}

Single crystals of the molecular complexes of 4,4'-di-tert-butyl2,2'-bipyridyl ( $1 \mathrm{~g}, 3.7 \mathrm{mmol}$ ) (dtBBP, 98\% Aldrich) with either bromanilic (1.1 g, $3.7 \mathrm{mmol}$ ) (BRA, $>98 \% \mathrm{TCI}$ ) or iodanilic acid (1.5 g, $3.7 \mathrm{mmol}$ ) (IA) were grown from a methanol solution $(250 \mathrm{ml})(98 \%)$ with the stoichiometric amounts of the components by a slow evaporation of the solvent at room temperature. Iodanilic acid (IA) was prepared from $p$-chloranil according to the literature. ${ }^{19}$ The purity and identity were checked by elemental analysis. The molecular mass and isotopic pattern observed in the mass spectrum correspond well to the molecular formula of iodanilic acid $\left(\mathrm{C}_{6} \mathrm{H}_{2} \mathrm{O}_{4} \mathrm{I}_{2}\right)$. The impurity content was found to be a few to $10 \%$. Analysis (\% calcd, \% found for $\mathrm{C}_{6} \mathrm{H}_{2} \mathrm{O}_{4} \mathrm{I}_{2}$ ): C (18.39, $18.80), \mathrm{H}(0.51,0.64)$. The composition of the synthesized compounds was identified by elemental analysis using a Vario FT III Element Analyzer on C, $\mathrm{H}$ and N (\% exp./theor.): $\mathrm{C}(50.32 / 50.90), \mathrm{H}(4.48 / 4.64), \mathrm{N}(4.87 / 4.95)$ and $\mathrm{C}(45.98 / 45.66)$, $\mathrm{H}(3.93 / 3.92), \mathrm{N}(4.16 / 4.24)$ for dtBBP.BRA and dtBBP.IA, respectively.

\subsection{Thermal analysis}

Differential scanning calorimetry (DSC) runs for dtBBP-IA were recorded with a Perkin Elmer DSC 8500 calorimeter in the temperature range between 310 and $350 \mathrm{~K}$ with a scanning rate of $20 \mathrm{~K} \mathrm{~min}{ }^{-1}$. The temperature was measured with the accuracy better than $0.1 \mathrm{~K}$ (relative error). Thermogravimetric analysis (TGA) and differential thermal analysis (DTA) were carried out on a Setaram SETSYS16/18 instrument between 290 and $700 \mathrm{~K}$ with a ramp rate in a nitrogen atmosphere. 
antiferroelectric phase
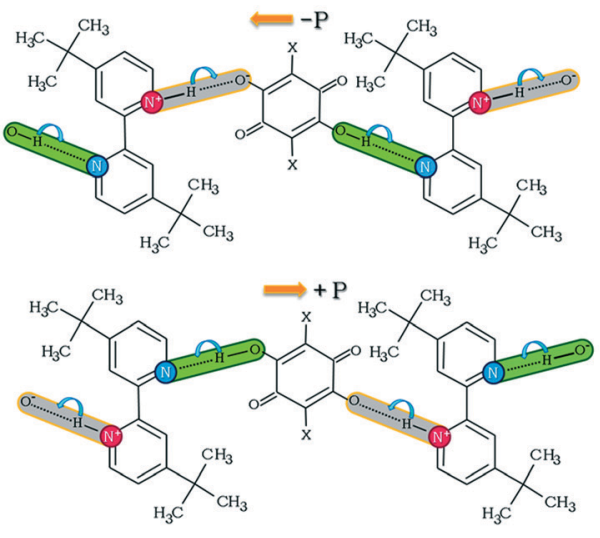

paraelectric phase

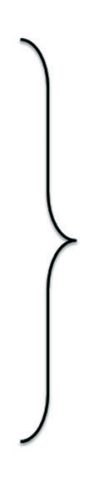

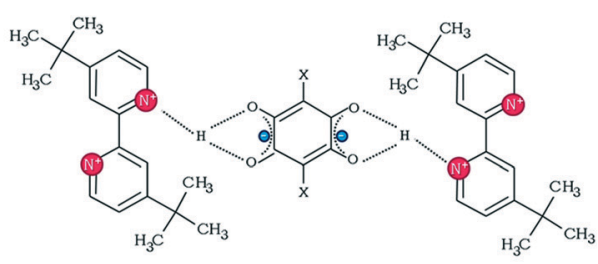

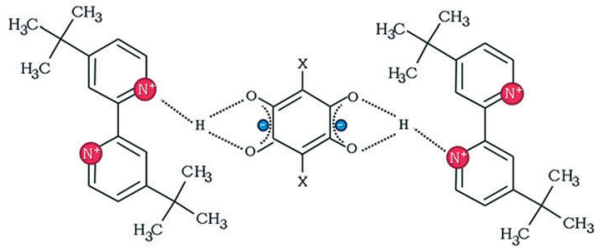

Scheme 1 Schematic diagram of the construction of the polar structure of the hydrogen-bonded chains consisting of the alternating monoprotonated dtBBP-base and $\pi$-molecular acid. On the right, the paraelectric phase is shown, where an intermediate structure between monovalent and divalent molecules is more likely.

\subsection{Single crystal X-ray diffractometry}

The X-ray diffraction data were collected using KUMA KM4CCD (dtBBP at 130 and $250 \mathrm{~K}$ and dtBBP.BRA) and Ruby $\mathrm{X}$-Calibur (dtBBP at $190 \mathrm{~K}$ and dtBBP-IA) $\kappa$-axis four circle diffractometers equipped with an Oxford Cryosystem cooler using graphite-monochromated MoK $\alpha$ radiation. The structures were solved by direct methods (SHELXS-2013) ${ }^{20}$ and refined by full-matrix least-squares on $F^{2}$ using the SHELXL2013 programs. ${ }^{21}$ All non-hydrogen atoms were refined with anisotropic thermal parameters. $\mathrm{H}$ atoms attached to $\mathrm{O}$ and $\mathrm{N}$ atoms were found in a difference Fourier map and refined with isotropic thermal parameters. Other $\mathrm{H}$ atoms were treated as riding and their isotropic temperatures were assumed as 1.2 and 1.5 times $U_{\text {eq }}$ of their closest heavy atoms. The crystal data together with experimental and refinement details are given in Table S2 (ESI $\dagger$ ). Full crystallographic data are deposited with the Cambridge Crystallographic Data Center (CCDC No. 1567116-1567121).

\subsection{Neutron scattering}

High resolution neutron spectra were measured on a SPHERES $^{22}$ backscattering spectrometer of the Jülich Centre for Neutron Science at Forschungsneutronenquelle Heinz Maier-Leibnitz FRM II (Technische Universität München (TUM), Garching) at temperatures between 100 and $300 \mathrm{~K}$ in the energy range of $\pm 30 \mu \mathrm{eV}$ with an energy resolution of about $0.65 \mu \mathrm{eV}$. The programs SLAW $^{23}$ and FRIDA $^{24}$ were used for reducing and fitting the data, respectively.

\subsection{Spectroscopic studies: IR and Raman}

Infrared spectra of dtBBP.IA were recorded in the temperature range between 298 and $330 \mathrm{~K}$ using a BRUKER IFS-88 FTIR spectrometer over the wavenumber range 4000-400 $\mathrm{cm}^{-1}$ with a resolution of $1 \mathrm{~cm}^{-1}$. The sample was prepared as a suspension in Nujol and embedded between $\mathrm{KBr}$ windows. An APD Cryogenics was used for temperature depen- dent studies in the temperature range between 300 and 350 K. FT-Raman spectra were measured with a FRA-106 attachment to the Bruker IF-88, having a resolution of $2 \mathrm{~cm}^{-1}$. The Nd:YAG laser power was ca. $200 \mathrm{~mW}$. The IR/Raman data were analysed by using the GRAMS/386 program from Galactic Industries.

\subsection{Dielectric measurements}

The complex dielectric permittivity, $\varepsilon^{*}=\varepsilon^{\prime}-i \varepsilon^{\prime \prime}$, has been calculated from the measured value of the complex admittance, $Y=G+i \omega C$, with an Agilent E4980A Precision LCR Meters in the frequency range between $100 \mathrm{~Hz}$ and $2 \mathrm{MHz}$ and in the temperature range between 100 and $350 \mathrm{~K}$. The dimensions of the samples were $5 \mathrm{~mm} \times 3 \mathrm{~mm} \times 1 \mathrm{~mm}$. As an electrode, a copper-foil was affixed to the crystals. The overall error in the estimation of the real and imaginary parts of the complex electric permittivity as well as of the conductivity was about $5 \%$. Dielectric measurements were carried out using a sample holder placed over liquid nitrogen vapour.

\section{$2.7{ }^{1} \mathrm{H}-\mathrm{NMR}$}

The NMR measurements were made using ELLAB TELAtomic PS 15 (operating at $25 \mathrm{MHz}$ in temperatures from room temperature down to the liquid nitrogen temperature) and Tecmag Scout (operating at $24.8 \mathrm{MHz}$ at temperatures below the liquid nitrogen temperature) spectrometers. Spinlattice relaxation times, $T_{1}$, were measured using a saturation sequence of $\pi / 2$ pulses followed by a variable time interval $\tau$ and a reading $\pi / 2$ pulse. The magnetization was found to recover exponentially within experimental error at all temperatures. The temperature of the sample down to the liquid nitrogen temperature was controlled by using a UNIPAN 660 temperature controller operating on a Pt 100 sensor providing long time temperature stability better than $1 \mathrm{~K}$. Below the liquid nitrogen temperature, a helium cooled Leybold cryostat was applied. Powdered samples of the neat dtBBP and its 
complexes with the organic acids were evacuated at room temperature and then sealed under vacuum in glass ampoules. All measurements were performed in a heating cycle. The errors in the measurements of $T_{1}$ were estimated to be about $5 \%$.

\section{Results}

\subsection{Thermal analysis}

By analyzing the results of the thermogravimetric measurements, one can state that the crystals of dtBBP, dtBBP.BRA and dtBBP.IA are stable up to $400 \mathrm{~K}$. In the temperature ranges up to the above-mentioned temperatures, the mass loss of the compounds is less than $1 \%$ (Fig. S1†).

The DSC results show that in the case of the pure base 4,4'-di-tert-butyl-2,2'-bipyridyl (dtBBP), two solid-to-solid phase transitions were observed in the temperature range between 300 and $100 \mathrm{~K}$ (Fig. S2a, ESI $\dagger$ ). Upon cooling, one peak at 216 $\mathrm{K}$ was found with enthalpy $(\Delta H)$ and entropy $(\Delta S)$ values of $1.7 \mathrm{~kJ} \mathrm{~mol}^{-1}$ and $7.9 \mathrm{~J} \mathrm{~K}^{-1} \mathrm{~mol}^{-1}$, respectively. While, upon heating, two peaks of thermal anomaly appeared, first at 165 $\mathrm{K}$ with $\Delta H=0.35 \mathrm{~kJ} \mathrm{~mol}^{-1}$ and $\Delta S=2.1 \mathrm{~J} \mathrm{~K}^{-1} \mathrm{~mol}^{-1}$, and the other one at $219 \mathrm{~K}$. The latter peak, at $219 \mathrm{~K}$, exhibits similar energy values to those found in the cooling cycle $(\Delta H=1.6 \mathrm{~kJ}$ $\left.\mathrm{mol}^{-1}, \Delta S=7.5 \mathrm{~J} \mathrm{~K}^{-1} \mathrm{~mol}^{-1}\right)$. The peak shape and the temperature hysteresis $(\Delta T=3 \mathrm{~K})$ present on the DSC curves clearly indicate the discontinuous character (first order) of the transition from the partially ordered (intermediate temperature, IT) to the disordered (high temperature, HT) phase. The single peak observed during the cooling cycle may be due to, on one hand, limitations of the applied method (the lowtemperature limit is $100 \mathrm{~K}$ ) or, on the other hand, the possibility that the low-temperature peak is not reversible.

In turn, in the case of the dtBBP.BRA complex, we have not found any indication in the DSC measurements that the crystals undergo a structural phase transition. In contrast to the complex described above, the reversible anomaly for dtBBP.IA at $331 \mathrm{~K}(\mathrm{I} \rightarrow \mathrm{II})$ (upon cooling/heating) has been detected. The two phases of dtBBP.IA are numbered I and II from the high to the low temperature one. The results of the calorimetric (DSC) measurements for dtBBP.IA are presented in Fig. S2b, ESI. $\uparrow$ The shape of the small thermal anomaly observed at $331 \mathrm{~K}$ is characteristic of a continuous (or second order) phase transition. The high ramp rate $\left(20 \mathrm{~K} \mathrm{~min}^{-1}\right)$ was used in order for the anomaly to be observed, because at ramp rates lower than the $20 \mathrm{~K} \mathrm{~min}^{-1}$ ramp rate, the peaks assigned to the phase transition were hardly observed.

\subsection{Crystal structure analysis}

The structural phase transitions found by the DSC method for 4,4-di-tert-butyl-2,2-bipyridyl (dtBBP) at 165 and $219 \mathrm{~K}$, and that for 4,4-di-tert-butyl-2,2-bipyridyl with iodanilic acid (dtBBP-IA) at $331 \mathrm{~K}$ as well as for the neat dtBBP and for comparison those found for dtBBP.CLA, ${ }^{17,18}$ are depicted in Scheme 2. This is the reason that the temperatures of the

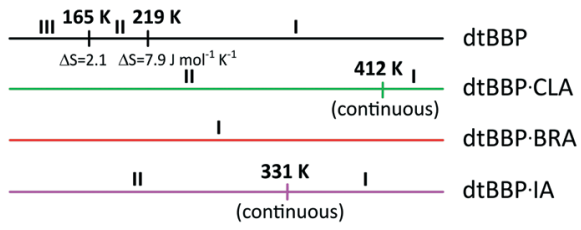

Scheme 2 The phase diagram for pure dtBBP and its molecular complexes with the halo-anilic acids.

X-ray measurements were chosen according to the different phases found for the single crystals under study.

The X-ray diffraction studies were carried out for the single crystals of dtBBP at 130 (phase III), 190 (phase II) and 250 $\mathrm{K}$ (phase I), for dtBBP.BRA at $155 \mathrm{~K}$ (no structural phase transitions found, phase I) and for dtBBP.IA at 295 (phase II) and $345 \mathrm{~K}$ (phase I). The asymmetric parts of the crystal structures for the phases analysed with the atom numbering are presented in Fig. 1.

dtBBP crystallizes in the monoclinic space group $P 2_{1} / c$ in all phases analysed. When we compare the low temperature phases with that at $250 \mathrm{~K}$, we can state that the unit cell is doubled in the phases II and III. At $250 \mathrm{~K}$, the asymmetric part of the unit cell consists of a half molecule of dtBBP. The tert-butyl groups are disordered, described by the two-site model with the occupation factor equal to $69: 31 \%$. The large ellipsoids of the thermal vibrations of the carbon atoms in the tert-butyl groups indicate the rotations of these groups around their $\mathrm{C}-\mathrm{C}_{(\text {ring) }}$ bonds. In the remaining two phases, II and III, the unit cell is doubled along the crystallographic $c$-axis, which is obviously accompanied by the doubling of the asymmetric part. Now, the whole dtBBP molecule becomes this asymmetric part. At $190 \mathrm{~K}$, we still deal with a disorder of the tert-butyl groups described by the one-site model with large thermal ellipsoids, which may indicate the diminishing of the freedom for rotations of these groups.
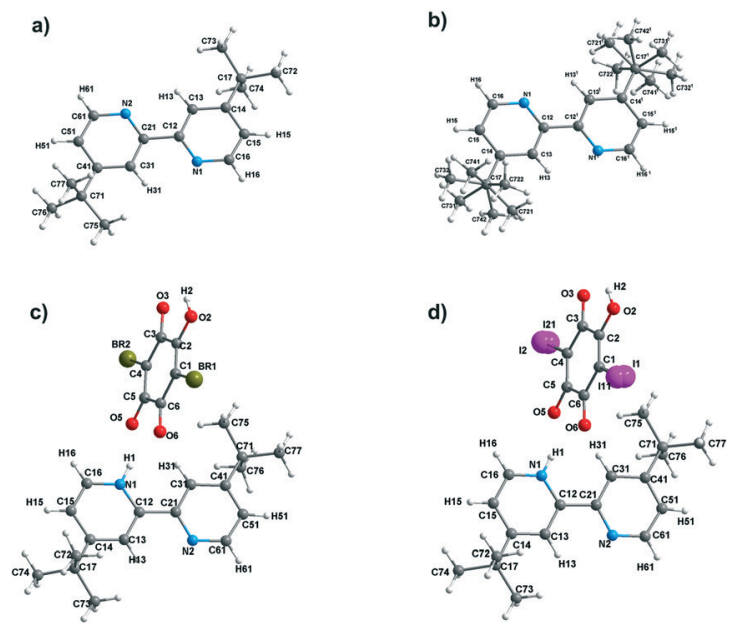

Fig. 1 The asymmetric parts with the atom numbering (a) for dtBBP at $130 \mathrm{~K}$ and (b) at $250 \mathrm{~K}$; (c) for dtBBP.BRA at $155 \mathrm{~K}$ and (d) for dtBBP.IA at $295 \mathrm{~K}$. 
The packing of the dtBBP molecules in the crystal structure at $130 \mathrm{~K}$ is presented in Fig. 2. The structures of all three phases are characterized by the existence of the layers of the dtBBP molecules. The molecules from two adjacent layers are connected by their tert-butyl groups. The layers are formed by the dtBBP molecules connected by weak $\mathrm{CH} \cdots \pi$ bonds. Each dtBBP molecule forms four $\mathrm{CH} \cdots \pi$ independent bonds, two as proton donors and two as acceptors (Fig. 3). The packing of the molecules and their mutual orientations are the same in all phases analysed. In turn, the ordering degree of the tert-butyl groups is different in the particular phases. This is probably a result of the strengthening of the molecular interactions between the adjacent layers in the structure on cooling the crystal.

In the case of the crystals of dtBBP with halo-anilic acids analysed in this work, the crystal structures are isostructural. In the case of dtBBP.IA, the iodide atoms are disordered, as described by the two-site model with the occupation factor equal to $54: 46 \%$ in both phases. The crucial element in their crystal structures is the infinite chains along the $b$-axis formed by the alternating dtBBP and acid molecules joined by the hydrogen bonds. The example of the chain in the dtBBP.BRA, the crystal formed by $\mathrm{N}-\mathrm{H} \cdots(\mathrm{O}, \mathrm{O})$ and $\mathrm{O}-\mathrm{H} \cdots(\mathrm{N}, \mathrm{O})$ bifurcated hydrogen bonds, is presented in Fig. S3 (ESI $\dagger$ ). The other characteristic feature of these crystals is the formation of the $\pi-\pi$ stacking structure. An example of the $\pi-\pi$ stacking in the crystal of dtBBP.BRA at $155 \mathrm{~K}$ is presented in Fig. S4 (ESI $\dagger$ ).

The selected most important bond lengths and angles for the crystalline state of the neat dtBBP and its complexes with the BRA, CLA and IA acids at different temperatures corresponding to the different phases of the particular crystals are

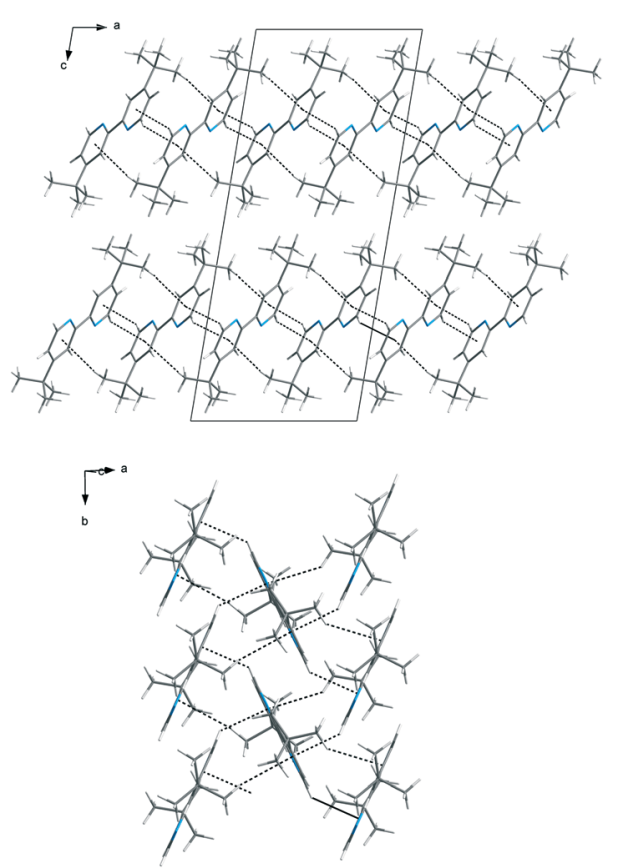

Fig. 2 The packing of the dtBBP in the crystal structure in phase III at $130 \mathrm{~K}$.

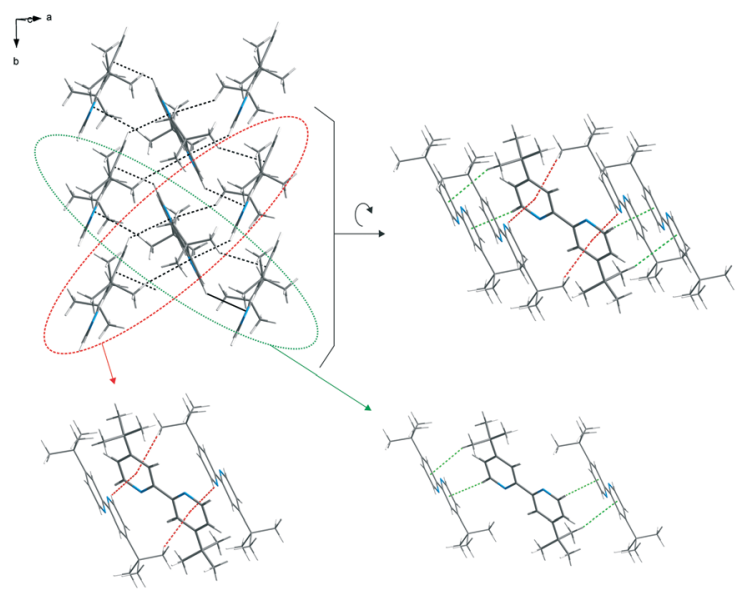

Fig. 3 The layers of the dtBBP molecules in the crystal structure at $130 \mathrm{~K}$ stabilized by weak $\mathrm{CH} \cdots \pi$ bonds (green and red lines).

collected in Tables S3 and S4 (ESI $\dagger$ ). The isomorphous crystal structures of the molecular complexes will be described on the basis of dtBBP.BRA. In the complex dtBBP.BRA, one hydrogen atom of the BRA hydroxyl group is transferred to the dtBBP moiety to form a $\mathrm{BRA}^{-}$anion and a $\mathrm{dtBBPH}^{+}$cation. Similar to that in the case of the dtBBP.CLA complex (see Table S5, ESI $\dagger$ ), the proton transfer in the hydrogen bond substantially shortens the corresponding $\mathrm{C}-\mathrm{O}$ bond in the BRA acid molecule (from 1.3264(4) to 1.261(4) $\AA$ ) and widens the $\mathrm{C}=\mathrm{N}-\mathrm{C}$ angles (from $116.9(3)$ to $122.0(3)^{\circ}$ ) in the dtBBP base molecule. Similar observations were present in the case of 5,5 - or 6,6'-dimethyl-2,2'-bipyridine with anilic acid., ${ }^{9,25}$ Studies of the single-crystal structures of, e.g. pentachlorophenol, with amines also prove that the $\mathrm{C}-\mathrm{O}$ bond length can be used to determine the ionic state of the complex. ${ }^{26}$ When the phenol $\mathrm{C}-\mathrm{O}$ distance is $\sim 1.36 \AA$, neutral phenol is observed, while the phenolic anion is formed for distances shorter than $1.26 \AA$.

The acid BRA and dtBBP base molecules are linked by bifurcated hydrogen bonds - $\mathrm{N}(1)-\mathrm{H}(1) \cdots \mathrm{O}(5)$ and $\mathrm{N}(1)-$ $\mathrm{H}(1) \cdots \mathrm{O}(6)$ as well as by the $\mathrm{O}(2)-\mathrm{H}(2) \cdots \mathrm{N}(2) \# 1$ hydrogen bond - to form a chain running along the $b$-axis. The corresponding $\mathrm{N}(1)-\mathrm{H}(1) \cdots \mathrm{O}(5), \mathrm{N}(1)-\mathrm{H}(1) \cdots \mathrm{O}(6)$ and $\mathrm{O}(2)-$ $\mathrm{H}(2) \cdots \mathrm{N}(2) \# 1$ distances are equal to 2.629(4), 2.972(4), 2.758(4) A, respectively. Moreover, the crystal packing is stabilized by the $\mathrm{C}-\mathrm{H} \cdots \mathrm{O}$ interactions as well as by the $\pi-\pi$ stacks (Table S6 and Fig. S4, ESI $\dagger$ ). They form with the bromanilic acid plane an angle of $73.24^{\circ}$. Also, in the crystal lattice, three contacts - $\mathrm{C}(72)-\mathrm{H}(722) \cdots \mathrm{O}(3) \# 4 \quad(3.456(5) \quad \AA), \quad \mathrm{C}(74)-$ $\mathrm{H}(743) \cdots \mathrm{O}(5) \# 5 \quad(3.421(5) \quad \AA)$ and $\mathrm{C}(76)-\mathrm{H}(61) \cdots \mathrm{O}(6) \# 7$ $(3.400(4) \AA)$ - are shorter than the sum of the van der Waals radii.

The crystal structures of dtBBP.IA were determined in two phases - I (345 K) and II (295 K). The structure at $295 \mathrm{~K}$, as it was mentioned above, is isostructural with those of the molecular complexes investigated in this paper. We have again the $[\mathrm{A} \cdots \mathrm{B} \cdots \mathrm{A} \cdots \mathrm{B}]_{\infty}$ infinite chains with the hydrogen bonds of either $\mathrm{O}-\mathrm{H} \cdots \mathrm{N}$ or $\mathrm{N}^{+}-\mathrm{H}^{\cdots} \mathrm{O}^{-}$types (see Fig. S5, and Table 
S7, ESI $\dagger$ ). Moreover, we can distinguish the directions of the chains according to the proton positions in the hydrogen bonds in adjacent chains. The crystal structure of dtBBP.IA is characterized by stacks of dtBBP molecules. The corresponding centroid to centroid distances in the dtBBP.XA $(\mathrm{X}=\mathrm{CL}$, BR, I) complexes are equal to CLA: 3.672 and $3.652 \AA$ A, BRA: 3.706 and $3.666 \AA$, IA: 3.910 and $3.823 \AA$. The molecules forming the stacks belong to the adjacent chains of hydrogen bonds. In this way, the hydrogen bond and the $\pi-\pi$ stacking interactions organize the described structure in the layers lying in the $b c$ plane according to a scheme (Scheme 3):

On the other hand, these layers stack one over the other along the $a$-direction. The weak interactions between layers are realized by the $\mathrm{H} \cdots \mathrm{H}$ contacts between methyl groups as well as by the $\mathrm{X} \cdots \mathrm{H}$ short contacts. In the low temperature phase of dtBBP.IA (below $331 \mathrm{~K}$ ), we deal with layers of the $[\mathrm{A} \cdots \mathrm{B} \cdots \mathrm{A} \cdots \mathrm{B}]_{\infty}$ chains in the $b c$ plane which are oriented in the same way along the $b$-axis. In a single layer, the chains are alternately oriented along one direction and in the adjacent layers they are parallel (see Fig. S6, ESI $\dagger$ ). In the high temperature phase (above $331 \mathrm{~K}$ ), the organization of the chains in one layer is the same as that in the LT phase. The mutual orientation of the chains in the adjacent layers is, however, changed. The chains in the adjacent layers become oriented antiparallel. It looks as if they are shifted by $0.5 c$ to each other. As a result, in the phase transition, the orientations of the chains change in every second layer in Fig. 4.

\subsection{Dielectric response}

Fig. 5 presents the temperature and frequency dependence of the real part of the electric permittivity of the pure dtBBP upon the cooling cycle. At temperatures close to $216 \mathrm{~K}$, the compound undergoes a phase transition from the disordered phase (HT) to the ordered phase (LT). This effect is observed as a small decrease of $\varepsilon^{\prime}$ near the $216 \mathrm{~K}$ phase transition. Due to the fact that the change of the crystal structure is related exclusively to an ordering of the tert-butyl/methyl groups, only a very small increment of the permittivity $(\Delta \varepsilon \cong 0.04)$ has been found at $216 \mathrm{~K}$.

Although no structural phase transition has been found for dtBBP-BRA, the measurements of the electric permittivity in a temperature range between 380 and $430 \mathrm{~K}$ showed dielectric dispersion and absorption.

The temperature and frequency dependence of the real, $\varepsilon^{\prime}$, and imaginary, $\varepsilon^{\prime \prime}$, parts of the complex electric permittivity is depicted in Fig. 6 and Fig. S7 (ESI $\dagger$ ). The changes in the electric permittivity are similar to those observed in the low-

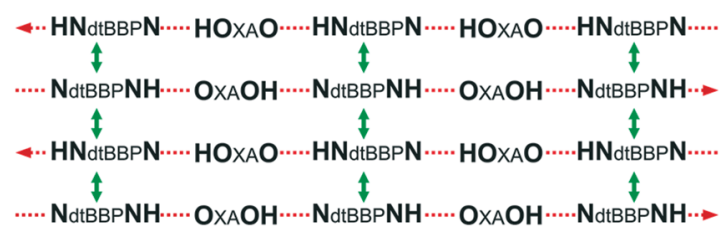

Scheme 3 The chains of the hydrogen bonds in dtBBP.XA.
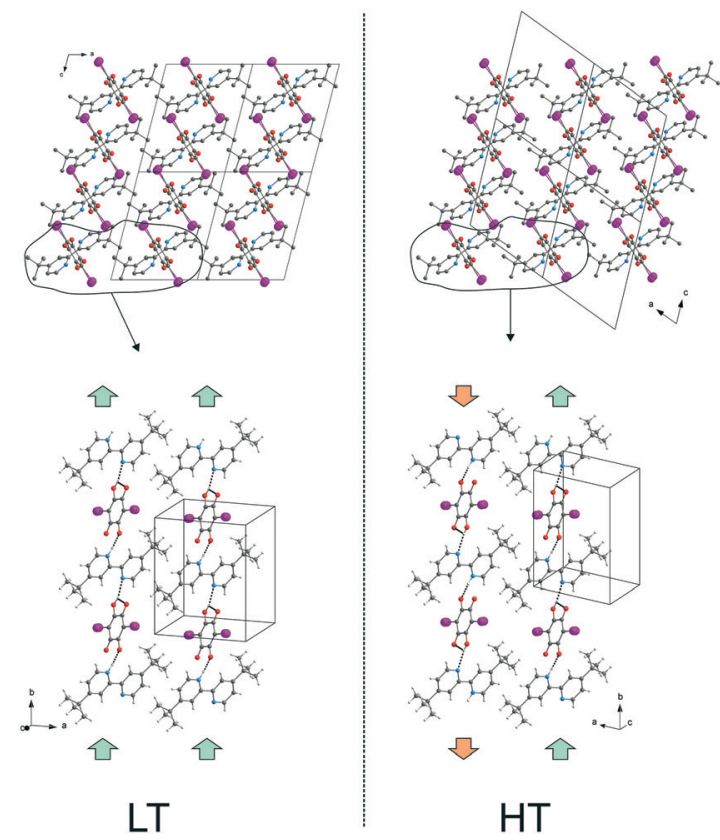

Fig. 4 The mutual orientation of the chains in the adjacent dtBBP.IA layers in the LT and HT phases.

temperature solid phase of the dtBBP.CLA crystal. ${ }^{14}$ We have analysed the results in terms of the Cole-Cole equation:

$$
\varepsilon^{*}=\varepsilon^{\prime}-i \varepsilon^{\prime \prime}=\varepsilon_{\infty}+\frac{\varepsilon_{0}-\varepsilon_{\infty}}{1+(i \omega \tau)^{1-\alpha}}
$$

where $\varepsilon_{\mathrm{o}}$ and $\varepsilon_{\infty}$ are the low and high-frequency limits, respectively, $\omega$ is the angular frequency, $\tau$ is the macroscopic relaxation time, and $\alpha$ represents the parameter of the relaxation time distributions.

Fig. S8 (ESI $\dagger$ ) shows the Argand diagrams at several temperatures for single crystals of dtBBP.BRA. At temperatures above $412 \mathrm{~K}$, the characteristic low-frequency-tail is observed, which is assigned to the ac conductivity of the sample. The same effect was obtained for dtBBP.CLA in the hightemperature phase (HT), also above 412 K. $^{17,18}$ From the

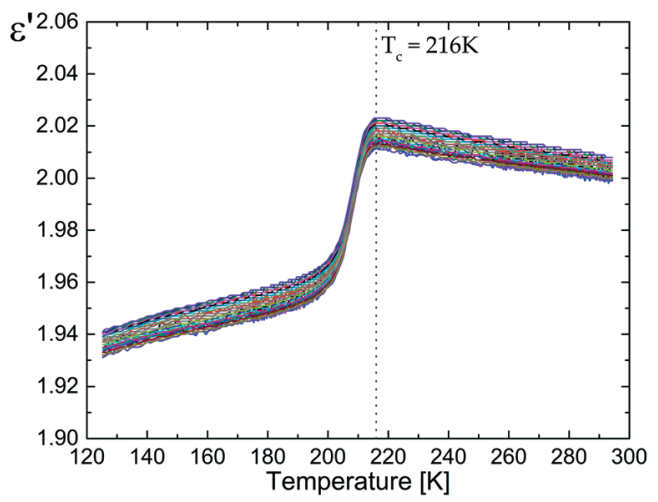

Fig. 5 Electric permittivity versus temperature measured for the polycrystalline pellets of the pure dtBBP (on cooling). 
a)

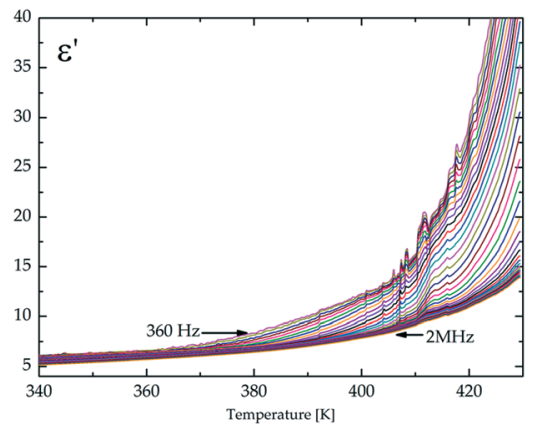

b)

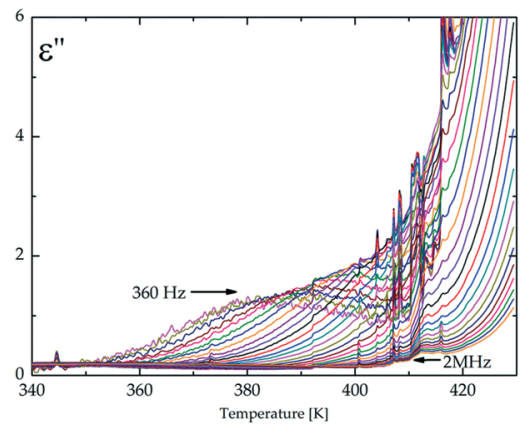

Fig. 6 The temperature dependence of (a) the real part, $\varepsilon^{\prime}$, and (b) the imaginary part, $\varepsilon^{\prime \prime}$; of the electric permittivity for the dtBBP.BRA complex along the $b$-axis.

Arrhenius relationship, $\tau=\tau_{\mathrm{o}} \exp \left(\frac{E}{R T}\right)$, where $\tau_{\mathrm{o}}$ is the relaxation time limit at high temperature, $E$ - activation energy, $R$ gas constant and $T$ - temperature, we have estimated the activation energy for the rotational process connected with the dielectric absorption to be equal to $108 \pm 5 \mathrm{~kJ} \mathrm{~mol}^{-1}$.

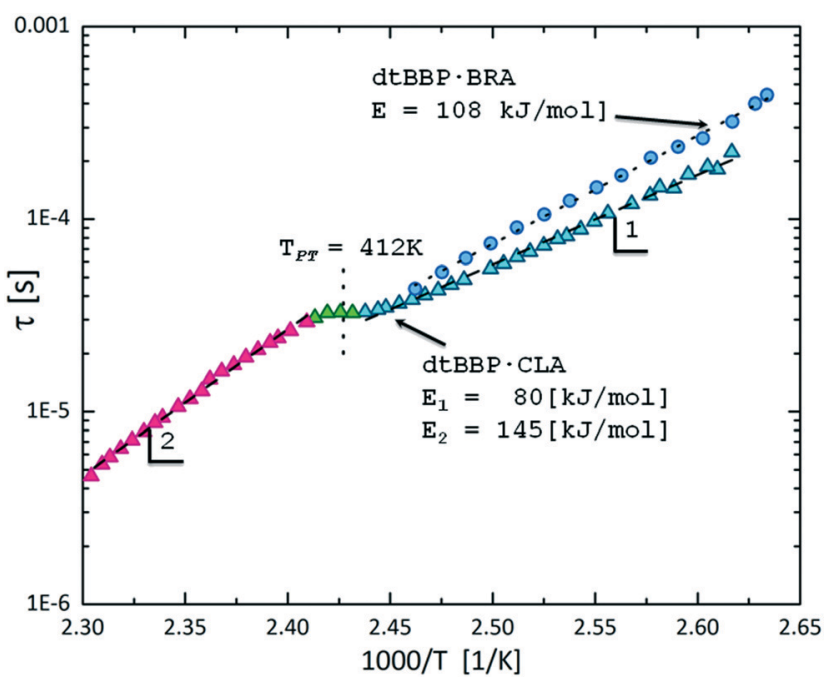

Fig. 7 The temperature dependence of the macroscopic dielectric relaxation time for dtBBP.CLA (triangles) ${ }^{17}$ and dtBBP.BRA (circles). The dtBBP.CLA low-temperature (LT) and high-temperature (HT) phases are indicated by 1 and 2, respectively.
Fig. 7 illustrates the comparison of the relaxation times for both dtBBP.CLA and dtBBP.BRA crystals, based on the Arrhenius dependence. Two activation energies were estimated for the dtBBP.CLA crystal, 80 and $145 \mathrm{~kJ} \mathrm{~mol}^{-1}$, for the lowtemperature phase (LT) and the high-temperature phase (HT), respectively, whereas in the case of the dtBBP-BRA molecular complex, the activation energy is equal to $108 \mathrm{~kJ}$ $\mathrm{mol}^{-1}$. This value is rather high for such a type of molecular motion and may indicate also a cooperative process, most probably related to the proton dynamics in the hydrogen bonds. As it was mentioned in the Introduction section, the dtBBP.CLA crystal undergoes one solid-to-solid phase transition at $412 \mathrm{~K}$. This transformation was also discussed in terms of the change in protons dynamics in the crystals. In the low-temperature (LT) phase, two adjacent polar chains of hydrogen bonds are created with opposite polarization directions, therefore, this phase has an antiferroelectric nature, as is shown in Scheme 1.

According to X-ray analysis, in both crystals (dtBBP.CLA and dtBBP.BRA), the molecules are monovalent, asymmetric and the ordered hydrogen bonds form a polar supramolecular chain. In the low-temperature phase of dtBBP.CLA and in the crystal of dtBBP.BRA, structural data indicate two types of hydrogen bonds: (i) one with proton transfer from an acid to a base $\left(\mathrm{NH}^{+} \cdots \mathrm{O}^{-}\right)$, and (ii) the other one with a hydrogen bond between a proton donor (CLA or BRA) and an acceptor (dtBBP). Analysis of the dielectric response and literature data allows us to conclude that the high-temperature (HT) phase for dtBBP.CLA is paraelectric. In the HT phase, the protons are localized as a superposition between nitrogen (from the base molecule) and two oxygen atoms from CLA. Both in the LT and HT phases, the proton transfer should be followed and the dynamical disorder of protons along the hydrogen chains generates the large relative permittivity in the vicinity of the phase transition. The mechanism of transformation was defined as the order-disorder and in the LT phase, the proton movements are in disorder, whereas the collective proton motions may be proposed in the HT phase.

We assumed that movement of the protons in the crystal of dtBBP.BRA could be the same like what was observed in the LT phase of the dtBBP with chloranilic acid (CLA). On the other hand, the crystal of dtBBP.IA also undergoes one phase transition at $331 \mathrm{~K}$, but in this case, we have observed only changes in the electrical permittivity in the close vicinity of the transition. It could be a consequence of the crystal preparation path; the iodanilic acid is unstable in solvents other than methanol. Crystallization in such a highly evaporating solvent causes the crystals to be of very poor quality and the electrical measurement was scrutinized on the powder sample (pellets). These results should be, therefore, treated only roughly.

\subsection{Infrared and Raman spectroscopy}

We have carried out the spectroscopic measurements in the temperature range between 11 and $300 \mathrm{~K}$ for dtBBP and between 303 and $353 \mathrm{~K}$ for dtBBP.IA in order to check if any 
changes in the IR spectra could be observed around their structural phase transitions (PT: dtBBP at 165 and 219 K, dtBBP.IA at $331 \mathrm{~K}$ ). Since the used wavenumber range corresponds to the internal vibrations of the organic molecules, any changes could indicate the possible mechanism of the PT. The measurements for both compounds were carried out on heating.

In Tables S8 and S9 (ESI $\dagger$ ), we have collected the wavenumbers of the observed bands in the IR spectra of dtBBP and dtBBP.IA and their tentative assignments to the internal vibrations of molecules. The assignments of the bands observed to the corresponding internal vibrations of dtBBP and dtBBP.IA were done on the basis of the corresponding literature. ${ }^{27-30}$

IR spectra of the polycrystalline dtBBP (at 11 and $300 \mathrm{~K}$ ) and dtBBP.IA (at 300 and $353 \mathrm{~K}$ ) as a suspension in Nujol and the FT-Raman spectra $(300 \mathrm{~K})$ between 3500 and 400 $\mathrm{cm}^{-1}$ are shown in Fig. S9a and b (ESI $\dagger$ ), respectively. For both compounds, only very small changes with temperature could be found. The following figures show the most spectacular changes in the temperature dependent IR spectra for dtBBP and dtBBP.IA, respectively.

Fig. 8 presents the temperature evolution of the bands in the IR spectra of dtBBP between 3090 and $3040 \mathrm{~cm}^{-1}$ and the temperature dependence of their respective wavenumbers. This wavenumber region corresponds to the stretching $\mathrm{C}-\mathrm{H}$ vibrations, $v(\mathrm{CH})$. In the low temperature phase of dtBBP, the band at $3069 \mathrm{~cm}^{-1}$ slightly diminishes its wavenumber and a step-wise change is observed at the $219 \mathrm{~K}$ PT temperature. In the high temperature, the wavenumber insignificantly increases on heating. The little effect of the PT can be observed also for the change in position at $165 \mathrm{~K}$.

In the wavenumber, which corresponds to out of plane C-H vibrations $\left(870-820 \mathrm{~cm}^{-1}\right)$, two bands are observed at 849 and $839 \mathrm{~cm}^{-1}$ (Fig. 9). Their relative intensities change with temperature: the high-wavenumber band becomes stronger and stronger. Moreover, their positions change in the PT at $219 \mathrm{~K}$, which is depicted in Fig. 9b and they are more spectacular again for the band at $849 \mathrm{~cm}^{-1}$.
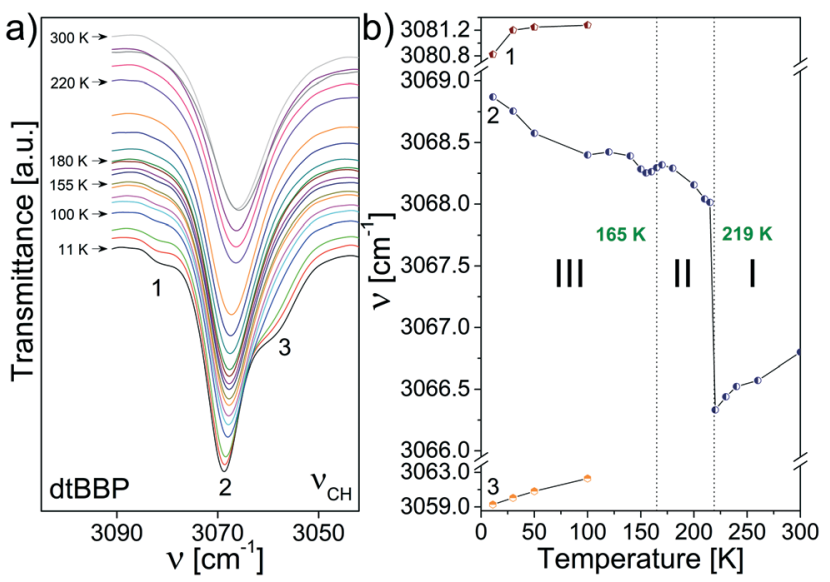

Fig. 8 (a) The temperature evolution of the bands assigned to the stretching $\mathrm{C}-\mathrm{H}$ vibrations, $v(\mathrm{CH})$, of dtBBP between 3090 and 3040 $\mathrm{cm}^{-1}$; (b) temperature dependencies of the band positions.
In turn, a phase transition has been found for dtBBP.IA at $331 \mathrm{~K}$. The extremely small shifts were found for the bands assigned to in-plane ring vibrations between 1700 and 1590 $\mathrm{cm}^{-1}$ (Fig. 10). In this wavenumber region, three well separated bands are observed (at 1664, 1623 and $1598 \mathrm{~cm}^{-1}$ ). The medium wavenumber band increases its wavenumber at the PT, and the other two bands decrease.

Similar to that for the neat dtBBP, changes in positions have been found for the bands assigned to the out of plane $\mathrm{C}-\mathrm{H}$ vibrations, which is presented in Fig. 11. The largest changes are observed at the $331 \mathrm{~K}$ phase transitions and the bands lay closer and closer one to another in the high temperature phase.

On the basis of the presented results of the temperature dependent IR spectra, the mechanisms of the structural phase transitions at $219 \mathrm{~K}$ of dtBBP and at $331 \mathrm{~K}$ of dtBBP.IA may be connected with the change in the dynamical state of the tert-butyl groups.

\subsection{Quasielastic neutrons scattering (QENS)}

QENS signals appear in the neutron scattering spectra, which concern the almost elastic collisions of neutrons with atoms. As a consequence, a broadening of the elastic line in the neutron energy spectrum, $S(\omega, \Omega)(\Omega$ - momentum and $\omega$ - energy transfer), is observed. The elastic scattering of neutrons is due to the atoms, which move too slowly to be resolved with the resolution of the instrument and move in a stochastic manner. ${ }^{31}$ The QENS signal contains both coherent and incoherent scattering contributions. The former process yields information about interference phenomena among atoms, like collective dynamics or transport properties, and it depends on the momentum transfer, $\Omega$. The latter one - the incoherent scattering - comes from individual atoms and can give information about the single-particle motions. It does not depend on $\Omega .^{32}$ At low temperatures, the molecular rotations or long-range diffusions are almost frozen but the dynamics of the methyl
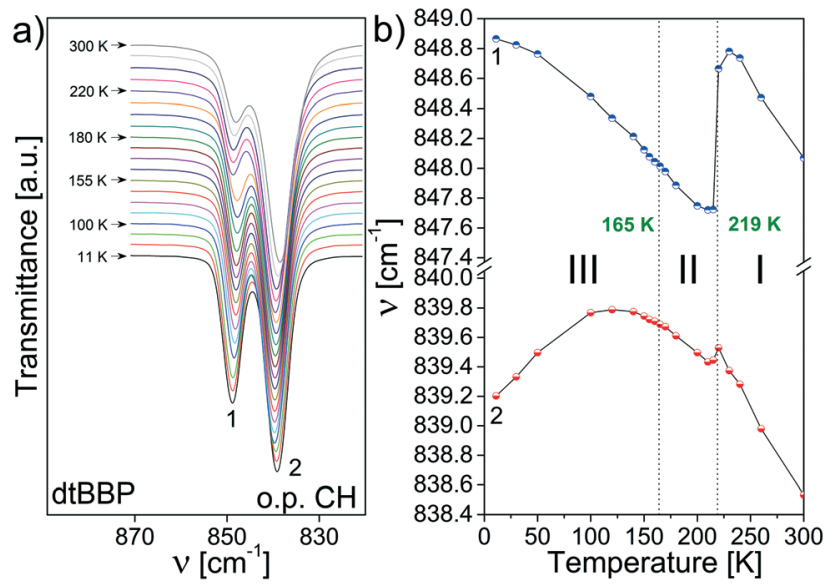

Fig. 9 (a) The bands at 849 and $839 \mathrm{~cm}^{-1}$ (RT) in the dtBBP spectra assigned to the out of plane $\mathrm{C}-\mathrm{H}$ vibrations; (b) temperature dependencies of their positions. 

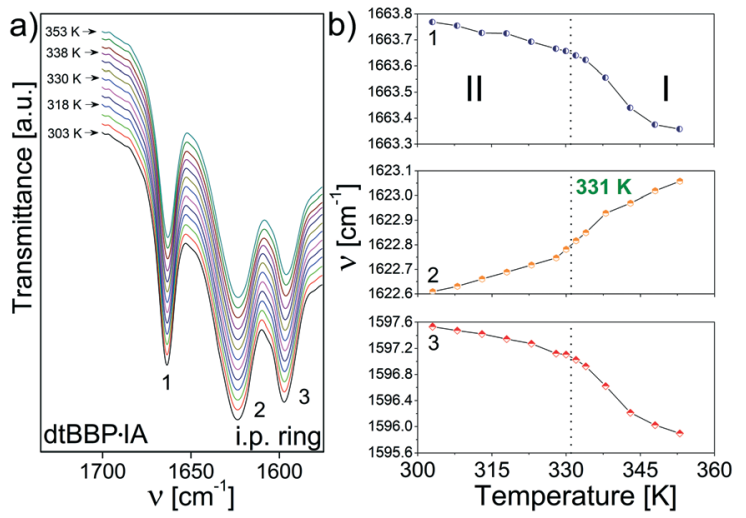

Fig. 10 (a) The temperature evolution of the bands assigned to dtBBP.IA in-plane ring vibrations between 1700 and $1590 \mathrm{~cm}^{-1}$, (b) temperature dependencies of their positions.
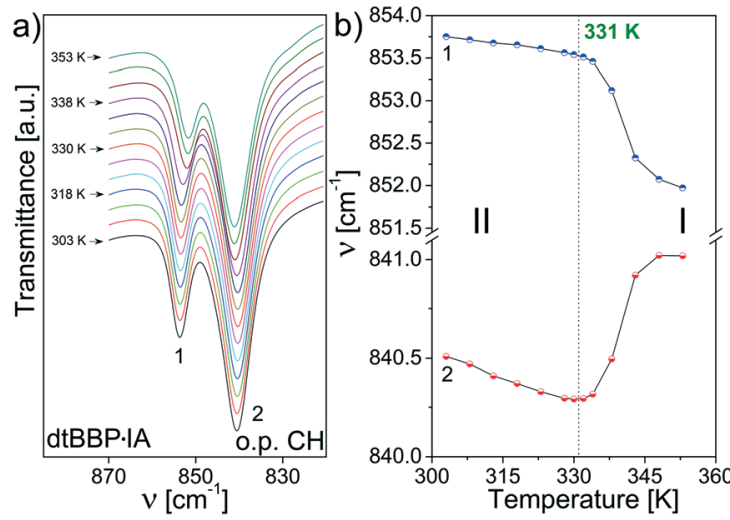

Fig. 11 (a) The bands assigned to the out of plane $\mathrm{C}-\mathrm{H}$ vibrations (870$820 \mathrm{~cm}^{-1}$ ) for dtBBP.IA; (b) temperature dependencies of their positions.

groups can still be present. The quasi-elastic component, if it appears at all, will be observed at temperatures above $c a .50 \mathrm{~K}$ and the motion of methyl groups can be described by a classical hopping process. The classical jumps of the hydrogen atoms over the energy barrier can be postulated from the temperature dependent QENS broadening of the elastic line.

The spectra measured at several temperatures between 150 and $300 \mathrm{~K}$ could be described by the instantaneous $120^{\circ}$ jumps of the $\mathrm{CH}_{3}$ groups around the three-fold symmetry axis with the scattering law $S(\omega, \Omega)$ given in ref. 33-35.

$$
\begin{aligned}
S(Q, \omega)= & \frac{1}{3}\left(1+\frac{2 \sin (Q r \sqrt{3})}{(Q r \sqrt{3})}\right) \delta(\omega) \\
& +\frac{2}{3 \pi}\left(1+\frac{2 \sin (Q r \sqrt{3})}{(Q r \sqrt{3})}\right) \frac{\Gamma}{\Gamma^{2}+\omega^{2}}
\end{aligned}
$$

where $r=1.06 \AA$ is the distance between protons in the rotated $\mathrm{CH}_{3}$ groups, and $\Gamma=\frac{3 \hbar}{2 \tau}$ is the bandwidth parameter, in which $\tau$ is the mean time between the proton jumps.
The QENS spectra of the pure base, dtBBP, and its molecular complexes with the organic acids, CLA, BRA and IA, are shown in Fig. 12. In the case of the complexes, only the protons from the $\mathrm{CH}_{3}$ groups of the dtBBP molecules take part in the fast reorientation described by the scattering function given above $\left(N_{\mathrm{q}}=18\right)$. The other protons from the dtBBP molecules and the organic acid ones contribute only to the elastic part of the spectrum $\left(N_{\mathrm{e}}=6\right)$. The solid lines represent the fittings to the QENS spectrum. The model used was convoluted with the resolution function. The instrumental resolution function was taken from the sample scattering at a temperature of $c a .4 \mathrm{~K}$, where only the elastic neutron scattering line was observed. The fitted mean time between jumps, $\tau$, changes for pure-dtBBP from $6.10 \times 10^{-8}(100 \mathrm{~K})$ to $6.10 \times$ $10^{-10} \mathrm{~s}(200 \mathrm{~K})$. For complexes with anilic acid, the $\tau$ changes as follows: (i) dtBBP.CLA from $4.12 \times 10^{-8}(150 \mathrm{~K})$ to $1.80 \times$ $10^{-9} \mathrm{~s}(250 \mathrm{~K})$, (ii) dtBBP.BRA from $6.20 \times 10^{-8}(150 \mathrm{~K})$ to 2.05 $\times 10^{-9} \mathrm{~s}(250 \mathrm{~K})$, and (iii) dtBBP.IA from $3.45 \times 10^{-8}(150 \mathrm{~K})$ to $1.85 \times 10^{-9} \mathrm{~s}(250 \mathrm{~K})$. The activation energy obtained from the Arrhenius plot $\ln \Gamma$ versus 1000/T (see Fig. S10, ESI $†$ ) changes from $80 \mathrm{meV}\left(\sim 8 \mathrm{~kJ} \mathrm{~mol}^{-1}\right)$ for pure dtBBP to $113 \mathrm{meV}(\sim 11$ $\mathrm{kJ} \mathrm{mol}^{-1}$ ) for the dtBBP.BRA complex. In fact, the activation energies obtained for the complexes are slightly higher than that obtained for the pure dtBBP. It means that the methyl groups rotate more freely in the pure base crystal than in those of the molecular complexes under study.

This observation is quite opposite to that observed for hexamethylbenzene (HMB) and its complex with tetracyanoquinodimethane (TCNQ) ${ }^{36}$ and for durene and the durene-TCNB complex ${ }^{37}$ as well as for TMP and the MPTCNB complex. ${ }^{38}$ For the latter compounds, it was found that the methyl groups rotated more freely in the crystals of the molecular complexes in comparison to those estimated for the pure crystals.

\section{6 ${ }^{1} \mathrm{H}$-NMR relaxation}

The temperature dependence of the ${ }^{1} \mathrm{H}$ NMR spin-lattice relaxation times $\left(T_{1}\right)$ for the studied compounds is shown in Fig. 13a and S11 (ESI $\dagger)$. The dependence of $T_{1}$ for the complexes of dtBBP with acids has two distinct ranges: the low temperature region with a plateau of $T_{1}$ and a high temperature one with the double minimum.

At low temperatures, below the liquid nitrogen temperature, the measured relaxation times form plateau with the nearly constant values of $T_{1}(1.7 \div 3.25 \mathrm{~s})$. This means that at these temperatures, the dominant mechanism of relaxation is related to the interactions of protons with the adjacent quadrupolar halogen atoms. Moreover, the single minima in the spin-lattice relaxation times have been detected for the complexes under study. We have attempted to fit these single minima taking into account the interactions of protons with neighbouring quadrupolar halogen nuclei $(\mathrm{Cl}, \mathrm{Br}) \cdot{ }^{39,40}$ The interactions are probably the main reason of the visible characteristic flattening of the spin-lattice relaxations time runs at low temperature. 
a)

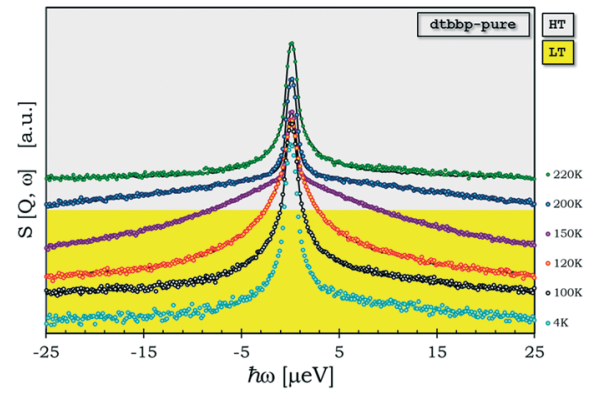

b)

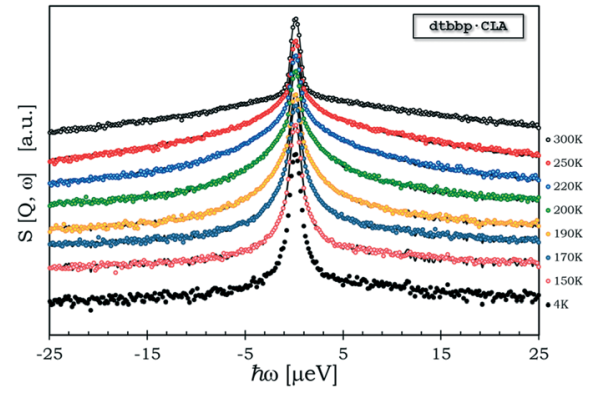

c)

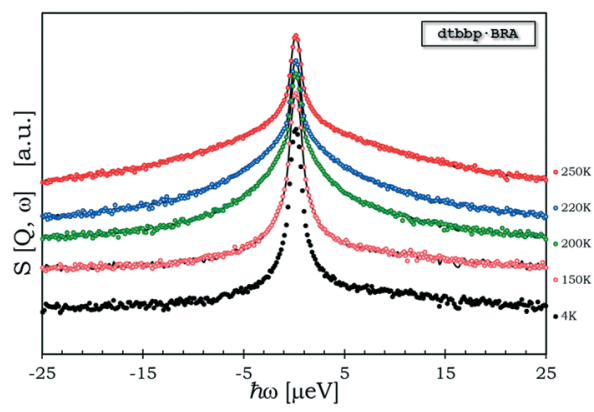

d)

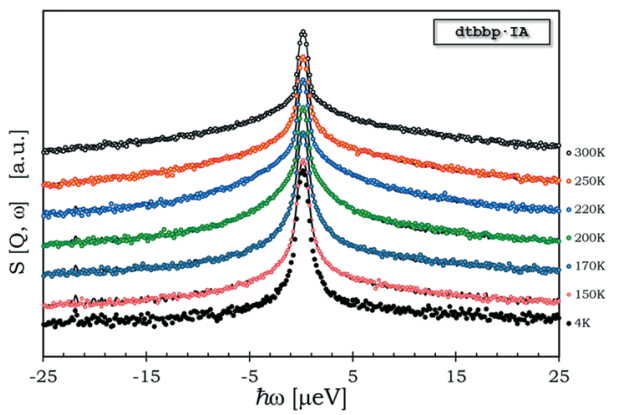

Fig. 12 Quasielastic spectra of: a) pure dtBBP, b) dtBBP.CLA, c) dtBBP.BRA and d) dtBBP.IA complexes. Solid lines are fitted with Dirac, Lorentzian components and resolution function obtained for samples at $4 \mathrm{~K}$.

The obtained theoretical fit curves were drawn with the quadrupolar spin-lattice rates of each halogen $\left(R_{\mathrm{Q}}^{\mathrm{Cl}}=5.7 \times\right.$ $10^{-9} \mathrm{~s}$ and $R_{\mathrm{Q}}^{\mathrm{Br}}=4.0 \times 10^{-9} \mathrm{~s}$ ) and with suitable relaxation constants $C_{\mathrm{HCl}}=3.1 \times 10^{6} \mathrm{~s}^{-2}$ and $C_{\mathrm{HBr}}=5.9 \times 10^{6} \mathrm{~s}^{-2}$. As usual, the quadrupolar relaxation has been assumed to be temperature independent. The contribution of the dipole-dipole cou- pling to the effective modulations of the proton-quadrupolar halogen interactions causes the proton relaxation time to appear to remain constant despite the fact that the correlation time, $\tau_{\mathrm{c}}$, becomes very long at low temperatures.

In the other temperature range, near the liquid nitrogen temperature, for each studied sample, the wide and double minimum has been observed with the following parameters: $T_{1 \mathrm{~min}}=15 \mathrm{~ms}$ at $105 \mathrm{~K}$ and $T_{1 \mathrm{~min}}=62 \mathrm{~ms}$ at $187 \mathrm{~K}$ for pure dtBBP, $T_{1 \min }=19 \mathrm{~ms}$ at $160 \mathrm{~K}$ and $T_{1 \min }=24 \mathrm{~ms}$ at $200 \mathrm{~K}$ for dtBBP.CLA, $T_{1 \mathrm{~min}}=26 \mathrm{~ms}$ at $155 \mathrm{~K}$ and $T_{1 \mathrm{~min}}=38 \mathrm{~ms}$ at 230 $\mathrm{K}$ for dtBBP.IA. The analysis of the double spin-lattice relaxation time minima according to the $\mathrm{BPP}^{41}$ theory requires that the sum of two different contributions has to be taken into account:

$$
\frac{1}{T_{1}^{\text {Total }}}=C_{1}\left(\frac{\tau_{1}}{1+\omega_{0}{ }^{2} \tau_{1}^{2}}+\frac{4 \tau}{1+4 \omega_{0}{ }^{2} \tau_{1}^{2}}\right)+C_{2}\left(\frac{\tau_{2}}{1+\omega_{0}{ }^{2} \tau_{2}^{2}}+\frac{4 \tau_{2}}{1+4 \omega_{0}{ }^{2} \tau_{2}^{2}}\right)
$$

where $C_{1}$ and $C_{2}$ are the motional constants, $\tau_{1}$ and $\tau_{2}$ - the correlation times for each component, respectively, and $\omega_{\mathrm{o}}-$ the Larmor frequency. Fig. S11 (ESI $\dagger$ ) contains the theoretical lines obtained from the fitting procedure using eqn (3). The estimated parameters in eqn (3) are collected in Table S10. $\dagger$ It should be noted that both relaxation constants $C_{i}$ contain the suitable fraction of the appropriate magnetization. The obtained parameters $C_{i}$ show similar proportions for all compounds analysed: $C_{1}: C_{2}=2: 1$, in which for every sample the low temperature relaxation component is greater than the high temperature one. Moreover, for the complexes of dtBBP with acids at high temperatures, we have found the rather high values of the negative index of the correlation times for the relaxing component. This means that the relaxation times found for the $\mathrm{dtBBPH}^{+}$cations are similar to those estimated for the similar molecules. ${ }^{42,43}$

For neat dtBBP, the temperature dependence of the second moment, $M_{2}$, of the proton NMR line (Fig. 13b) has been measured between $96 \mathrm{~K}$ and $293 \mathrm{~K}$. On heating from $96 \mathrm{~K}$, the second moment decreases from about $8 \mathrm{G}^{2}$ to about $3 \mathrm{G}^{2}$ around $140 \mathrm{~K}$. Then, up to room temperature, the values of the second moment remain nearly constant. The theoretical calculations of the second moment were carried out according to the van Vleck method ${ }^{44}$ for the rigid state of dtBBP, basing on the structural crystallographic data with an assumed distance between carbon and hydrogen atoms, $\mathrm{C}-\mathrm{H}$, equals $1.09 \AA$. The calculations lead to a value of $M_{2}^{\text {Rigid }}$ equals $21.88 \mathrm{G}^{2}$. This result means that such a frozen state of the analysed compound has not been reached. The characteristic bend of the reduction of the second moment value just above $96 \mathrm{~K}$ suggests that the whole narrowing of the proton NMR line should undergo in two steps. It was impossible to carry out the measurements of $M_{2}$ at low temperatures due to the limitation of the cooling system, so that, regretfully, the first step has not been detected. Nevertheless, the theoretical dashed line as shown in the Fig. 13b is perfectly consistent 
a)

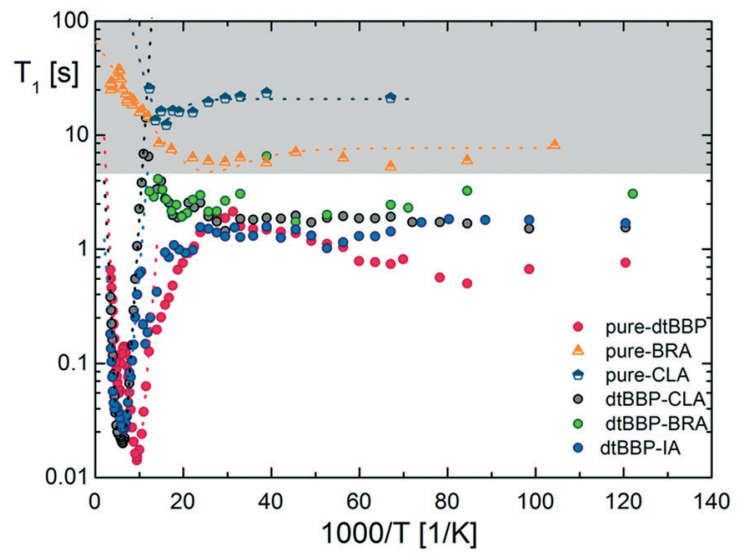

b)

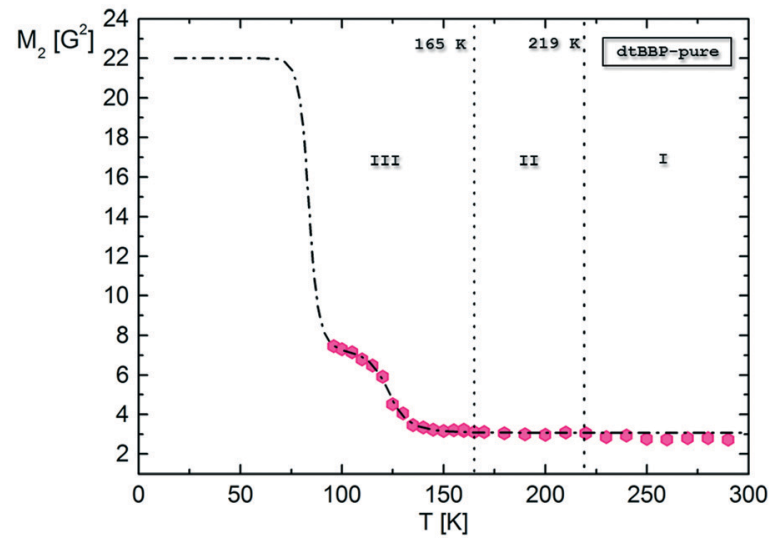

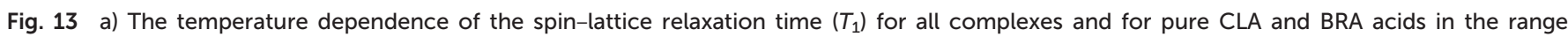
between 8 and $100 \mathrm{~K}$, and b) the temperature dependence of the second moment, $M_{2}$, for neat dtBBP

with the experimental results of the other reduction of $M_{2}$ in the vicinity of $100 \mathrm{~K}$. The similar behaviour of the second moment has been found in the other compounds analysed by us. ${ }^{45,46}$ In the latter papers, the way of the fitting procedure for the $M_{2}$ temperature runs was described in detail. For this reduction, the dynamical parameters obtained are the following: activation energy $E_{\mathrm{a}}=4.3 \mathrm{kcal} \mathrm{mol}^{-1}\left(18 \mathrm{~kJ} \mathrm{~mol}^{-1}\right)$ and correlation time $\tau_{c 0}=4.3 \times 10^{-13} \mathrm{~s}$. It should be noted that the expected value of $M_{2}$ at low temperatures, i.e. $23 \mathrm{G}^{2}=$ $21.88 \mathrm{G}^{2}$ plus inter component, reduces at high temperature to nearly a quarter of its primary value, which additionally confirms our estimation.

\section{Discussion and conclusions}

In this paper, we have described the properties of pure base 4,4'-di-tert-2,2'-bipyridyl (dtBBP) and its two molecular complexes containing two different organic acids: bromanilic (dtBBP-BRA) or iodanilic (dtBBP-IA) acid. The main purpose of this work was the detailed analysis of the intermolecular interactions in the solid state and their effect on the macroscopic properties of the crystals. The experimental results are compared with those of the chloranilic analogue (dtBBP.CLA). ${ }^{17,18}$

The single-crystal X-ray diffraction studies reveal that all complexes of dtBBP with halo-anilic acids described in this paper crystallize in the 1:1 stoichiometry. We have found that both crystals of dtBBP-BRA and dtBBP.IA show a layer structure. The molecular organization within the layer is governed by two types of the intermolecular interactions. First interactions are related to the $\mathrm{OH} \cdots \mathrm{N}$ hydrogen bonds and the other ones to $\pi-\pi$ stacking which connects chains into layers. The crystal packing of the pure dtBBP can be described by the layer structure as well. The interactions exhibit, however, a different nature (dispersive).

In both complexes with anilic acids, we deal with the proton transfer in the ${ }^{-} \mathrm{O} \cdots \mathrm{HN}^{+}$hydrogen bond (h.b.) on one side of the dtBBP molecule. On the other side of dtBBP, we deal with the classical h.b. without proton transfer. The evidence of the $\mathrm{dtBBPH}^{+}$protonation includes both the shortening of the $\mathrm{C}(5)-\mathrm{O}(5)$ distances from 1.326 to $1.261 \AA$ in $\mathrm{BRA}^{-}$ or from 1.331 to $1.260 \AA$ in $\mathrm{IA}^{-}$, and the enlargement of the $\mathrm{C}(16)-\mathrm{N}(1)-\mathrm{C}(12)$ angle from 116.9 to $122.0^{\circ}$ and from 116.5 to 121.8 , for dtBBP.BRA and dtBBP.IA, respectively.

Using thermal methods, the structural phase transitions were disclosed for the pure-dtBBP base and its complex with iodanilic acid (dtBBP.IA). For the dtBBP.BRA complex, no phase transition has been found. Then the phase diagram has been constructed (see Scheme 2).

In the case of the pure base, dtBBP, upon cooling, one solid-to-solid phase transition was observed, at $216 \mathrm{~K}$, while upon heating, two PTs appeared, at $165 \mathrm{~K}$ and $219 \mathrm{~K}$. In all phases analysed, of course, on heating from $130 \mathrm{~K}$, (phase III, II and I at 130, 190 and $250 \mathrm{~K}$, respectively), space group $P 2_{1} / c$ is unchanged and the phases differ by the tert-butyl group ordering. This is accompanied by a doubling of the unit cell in comparison to that in phase I. In the intermediate phase, the freedom of the tert-butyl group changes gradually with temperature. In the lowest temperature phase III, both tert-butyl groups of the dtBBP molecule are ordered. The observed changes in the structure on one hand very weakly impact the dielectric response and, on the other hand, significantly affect the neutron scattering of dtBBP. The small dielectric increment may be explained by the very little change in the dipole moment of the molecule connected either with the methyl or tert-butyl group rotations.

The existence of the infinite hydrogen bonded chains in crystals favours the long range interactions, which may result in the collective proton jumps in the chain. In consequence, these crystals could reveal a large electric permittivity value as well as they could show polar properties at room temperature. The molecule of the dtBBP-base can be an excellent candidate for the creation of one-dimensional hydrogen ribbons with the dynamical disorder of protons. The comparison of 
dtBBP.CLA with dtBBP.BRA allows us to state that although the latter crystal does not exhibit any structural phase transition, the dielectric response also indicates the collective jump of the protons through the infinite hydrogen-bonded chains. The energy, which is necessary to activate the protons, is rather high $\left(108 \mathrm{~kJ} \mathrm{~mol}^{-1}\right)$. The question is why the exchange of the chlorine atoms by the bromine ones has such an impact on the crystal properties that no structural phase transition has been observed up to the decomposition of dtBBP-BRA. Relying on the crystallographic data, it is known that the adjacent polar chains in one layer are antiparallel in the dtBBP-BRA crystal structure as well. The reason why the phase transition is not observed in this case should be assigned to subtle changes in the geometrical parameters of the main hydrogen bonds. In the case of dtBBP with bromanilic acid, the length between donor $\mathrm{N}^{+}(1)$ and proton $\mathrm{H}(1)$ is much longer than that for the dtBBP.CLA complex, $1.30(7)$ and 1.05(2), respectively. The distance between proton $\mathrm{H}(1)$ and acceptor $\mathrm{O}(5)$ is shorter for the bromanilic analogue (1.39(7), 1.60(2)-CLA). These data and the angle $\angle$ (D...A) can suggest that the main hydrogen bond between the acid and the base in dtBBP.BRA is weaker than that in dtBBP.CLA. Most likely, the generally weaker interactions in the complex with bromanilic acid are the reason for any structural changes with temperature.

We did not succeed in obtaining the dtBBP.IA single crystals of good quality for dielectric measurements and, as a consequence, the results presented in Fig. S12 and S13 may be treated only qualitatively. Nevertheless, an increase of the real part of the complex electric permittivity of dtBBP.IA may indicate some proton motions in the h.b. in the vicinity of the structural phase transition at $331 \mathrm{~K}$. These motions might be connected with the reorganizations of the molecular chains. This of course weakly affects the motions of methyl/ tert-butyl groups.

The analysis of the QENS results over a broad temperature range allows us to evaluate the activation energy for the stochastic jumps of the methyl groups. In the temperature range between 150 and $300 \mathrm{~K}$, the QENS signal has been observed for neat dtBBP and its complexes with the organic acids, CLA, BRA and IA. The activation energies found are slightly higher for the complexes than those estimated for neat dtBBP. The differences in the activation energy are most probably due to the other contact lengths between the hydrogen atoms in tert-butyl groups and the adjacent molecules. This causes the higher barrier for the methyl/butyl rotations in the complexes. It should be noted that the effect of the charge transfer in these molecules seems to be negligible, as there are insignificant differences among the dtBBP complexes with different acids.

The gradual release of the rotational freedom proposed in the crystals of dtBBP is almost consistent with runs of the ${ }^{1} \mathrm{H}$ NMR second moment, $M_{2}$. Taking into account the temperature dependence both of the spin-lattice relaxation time, $T_{1}$, and $M_{2}$, we can assume that at low temperatures, $T<100 \mathrm{~K}$, the lattice of the dtBBP compounds is still not rigid. Below ca. $96 \mathrm{~K}$, we deal still with the methyl group rotations, whereas the whole tert-butyl groups are rigid. Above let say $100 \mathrm{~K}$, we can see the onset of the release of the whole tert-butyl group dynamics follows, which is manifested in the reduction of the $M_{2}$ value. From these $M_{2}$ runs it shows, however, that the tert-butyl group rotations begin already in phase III (below $165 \mathrm{~K}$ ).

The magnetization relaxation found for the complexes studied seems to be similar in nature to that in the case of the other molecular-ionic crystals investigated by us earlier. ${ }^{37,38}$ At high temperature, the double minimum on the $T_{1}$ runs for all three compounds, with the proportion $2: 1$, may suggest that at temperatures close to the liquid nitrogen temperature, methyl groups' non-equivalence in the crystal structure in this proportion is present.

The temperature dependent infrared spectra confirmed first of all the proton transfer in hydrogen bonds in the molecular complex of dtBBP.IA. We have observed the fingerprints of the $\mathrm{O}-\mathrm{H} \cdots \mathrm{N}$ hydrogen bond and the bands assigned to the vibrations of the $\mathrm{NH}^{+}$group ( $c a .2300 \mathrm{~cm}^{-1}$ ). As regards to the phase transitions found at 165 and $219 \mathrm{~K}$ for pure dtBBP and at $331 \mathrm{~K}$ for dtBBP.IA, only very small changes with temperature could be found. The most spectacular changes are observed for the bands assigned to the $\mathrm{C}-\mathrm{H}$ vibrations. This is consistent with the postulated changes in dynamics of the methyl/tert-butyl groups with temperature. Particularly the mechanism of the phase transition at $331 \mathrm{~K}$ for dtBBP.IA may affect very slightly the infrared spectra since it is connected with different organizations of the hydrogen bonded chains of molecules. This is also consistent with the observed dielectric response of the crystals.

In the case of the infrared spectra of the pure dtBBP, the changes are observed only around the high temperature phase transition at $219 \mathrm{~K}$ and they are undoubtedly connected with the change of the methyl/tert-butyl dynamics. The spectra were recorded for the suspensions in Nujol, which exhibit the bands overlapping those coming from the $\mathrm{OH}$ groups involved in the hydrogen bonds, so that no changes in the spectra could be observed in this region.

\section{Conflicts of interest}

There are no conflicts to declare.

\section{Acknowledgements}

The authors gratefully acknowledge the financial support provided by the JCNS to perform the neutron scattering measurements at the Heinz Maier-Leibnitz Zentrum (MLZ), Garching, Germany.

The author, Marcin Moskwa gratefully acknowledges the financial support provided by Wroclaw Centre of Biotechnology, programme The Leading National Research Centre (KNOW) for years 2014-2018. 
The paper is a result of the realization of the project No. UMO-2016/21/B/ST3/004640201/2078/17 financed by National Science Centre, Poland.

\section{References}

1 P. H. Stahl and C. G. Wermuth, Handbook of pharmaceutical salts: properties, selection and use; International Union of Pure and Applied Chemistry, Wiley-VCH, Weinheim, New York, 2002.

2 S. L. Johnson and K. A. Rumon, J. Phys. Chem., 1965, 69, 74-86.

3 A. J. Cruz-Cabeza, CrystEngComm, 2012, 14, 6362-6365.

4 K. Molčanov and B. Kojić-Prodić, CrystEngComm, 2010, 12, 925-939.

5 W. Sawka-Dobrowolska, G. Bator, B. Czarnik-Matusewicz, L. Sobczyk, A. Pawlukojć, E. Grech, J. Nowicka-Scheibe and $\mathrm{H}$. Rundlöf, Chem. Phys., 2006, 327, 237-246.

6 M. Prager, A. Wischnewski, G. Bator, E. Grech, A. Pawlukojć and L. Sobczyk, Chem. Phys., 2007, 334, 148-153.

7 A. Pawlukojć, M. Prager, W. S. Dobrowolska, G. Bator, L. Sobczyk, A. Ivanov, S. Rols, E. Grech, J. Nowicka-Scheibe and T. Unruh, J. Chem. Phys., 2008, 129, 154506.

8 J. Nowicka-Scheibe, E. Grech, W. Sawka-Dobrowolska, G. Bator, A. Pawlukojć and L. Sobczyk, J. Mol. Struct., 2010, 976, 30-35.

9 G. Bator, W. Sawka-Dobrowolska, L. Sobczyk, E. Grech, J. Nowicka-Scheibe, A. Pawlukojć, J. Wuttke, J. Baran and M. Owczarek, J. Chem. Phys., 2011, 135, 44509.

10 G. Bator, L. Sobczyk, W. Sawka-Dobrowolska, J. Wuttke, A. Pawlukojć, E. Grech and J. Nowicka-Scheibe, Chem. Phys., 2013, 410, 55-65.

11 G. Bator and L. Sobczyk, Trends Org. Chem., 2014, 17, 93-105.

12 S. Horiuchi, F. Ishii, R. Kumai, Y. Okimoto, H. Tachibana, N. Nagaosa and Y. Tokura, Nat. Mater., 2005, 4, 163-166.

13 S. Horiuchi, R. Kumai and Y. Tokura, J. Mater. Chem., 2009, 19, 4421.

14 J. Fujioka, S. Horiuchi, N. Kida, R. Shimano and Y. Tokura, Phys. Rev. B: Condens. Matter Mater. Phys., 2009, 80, 1-8.

15 K. Kobayashi, S. Horiuchi, S. Ishibashi, F. Kagawa, Y. Murakami and R. Kumai, Chem. - Eur. J., 2014, 20, 17515-17522.

16 S. Horiuchi and Y. Tokura, Nat. Mater., 2008, 7, 357-366.

17 M. Rok, A. Piecha-Bisiorek, P. Szklarz, G. Bator and L. Sobczyk, Chem. Phys., 2015, 452, 53-60.

18 G. Bator, W. Sawka-Dobrowolska, L. Sobczyk, M. Owczarek, A. Pawlukojć, E. Grech and J. Nowicka-Scheibe, Chem. Phys., 2012, 392, 114-121.

19 H. A. Torrey and W. H. Hunter, J. Am. Chem. Soc., 1912, 34, 702-715.

20 G. M. Sheldrick, Acta Crystallogr., Sect. A: Found. Crystallogr., 2007, 64, 112-122.

21 G. M. Sheldrick, Acta Crystallogr., Sect. C: Struct. Chem., 2015, 71, 3-8.
22 M. Zamponi and M. Khaneft, J. Large-scale Res. Facil., 2015, 1, A30.

23 J. Wuttke, SLAW - A neutron histogram to scattering law converter, http://apps.jcns.fz-juelich.de/slaw/.

24 J. Wuttke, FRIDA - Flexible rapid interactive data analysis, http://apps.jcns.fz-juelich.de/frida/.

25 S. Horiuchi, R. Kumai and Y. Tokura, Angew. Chem., Int. Ed., 2007, 46, 3497-3501.

26 S. L. Childs, G. P. Stahly and A. Park, Mol. Pharmaceutics, 2007, 4, 323-338.

27 M. A. Slifkin and B. M. Smith, Spectrochim Acta A, 1969, 25, 1479-1489.

28 P. K. Kipkemboi, P. P. Kiprono and J. J. Sanga, Bull. Chem. Soc. Ethiop., 2003, 17, 211-218.

29 E. Castellucci, L. Angeloni, N. Neto and G. Sbrana, Chem. Phys., 1979, 43, 365-373.

30 K. Łuczyńska, K. Drużbicki, K. Lyczko and J. C. Dobrowolski, J. Phys. Chem. B, 2015, 119, 6852-6872.

31 M. Karlsson, Phys. Chem. Chem. Phys., 2015, 17, 26-38.

32 M. Kofu, M. Tyagi, Y. Inamura, K. Miyazaki and O. Yamamuro, J. Chem. Phys., 2015, 143, 234502.

33 T. Springer, Quasielastic Neutron Scattering for the Investigation of Diffusive Motions in Solids and Liquids, Springer-Verlag, Berlin Heidelberg New York, 1972, vol. 64.

34 J. D. Barnes, J. Chem. Phys., 1973, 58, 5193.

35 M. Bee, Quasielastic Neutron Scatering, Adam Hilger, Bristol and Philadelphia, 1988.

36 W. Sawka-Dobrowolska, G. Bator, L. Sobczyk, A. Pawlukojć, H. Ptasiewicz-Bak, H. Rundlöf, J. Krawczyk, M. NowinaKonopka, P. Jagielski, J. A. Janik, M. Prager, O. Steinsvoll, E. Grech and J. Nowicka-Scheibe, J. Chem. Phys., 2005, 123, 1243305.

37 G. Bator, L. Sobczyk, A. Pawlukojć, J. Nowicka-Scheibe, E. Grech, J. Krawczyk, M. N. Konopka, I. Natkaniec, I. V. Kalinin and O. Steinsvoll, Phase Transitions, 2007, 80, 489-500.

38 J. Krawczyk, M. Nowina Konopka, J. A. Janik, O. Steinsvoll, G. Bator, A. Pawlukojć, E. Grech, J. Nowicka-Scheibe and L. Sobczyk, Collect. Czech. Chem. Commun., 2009, 74, 73-84.

39 J. Przesławski, W. Medycki, A. Piecha, R. Jakubas and D. Kruk, Chem. Phys., 2013, 410, 19-24.

40 M. Węcławik, P. Szklarz, W. Medycki, R. Janicki, A. PiechaBisiorek, P. Zieliński and R. Jakubas, Dalton Trans., 2015, 44, 18447-18458.

41 N. Bloembergen, E. M. Purcell and R. V. Pound, Phys. Rev., 1948, 73, 679-712.

42 M. Węcławik, A. Gagor, A. Piecha, R. Jakubas and W. Medycki, CrystEngComm, 2013, 15, 5633.

43 M. Wojciechowska, P. Szklarz, A. Białońska, J. Baran, R. Janicki, W. Medycki, P. Durlak, A. Piecha-Bisiorek and R. Jakubas, CrystEngComm, 2016, 18, 6184-6194.

44 J. H. Van Vleck, Phys. Rev., 1948, 74, 1168-1183.

45 P. Szklarz, J. Zaleski, R. Jakubas, G. Bator, W. Medycki and K. Falińska, J. Phys.: Condens. Matter, 2005, 17, 2509-2528.

46 W. Medycki and R. Jakubas, Solid State Nucl. Magn. Reson., 1999, 15, 73-77. 